\title{
Teaching Approach and Materials for Enhancing Student Learning Experience in Tomography Engineering: Soft-Field Tomography
}

\author{
Wuliang Yin ${ }^{1,2^{*}}$, Trevor York ${ }^{2}$, Krikor B. Ozanyan ${ }^{2}$ \\ ${ }^{1}$ College of Electronic Information and Automation, Tianjin University of Science and Technology, Tianjin, China \\ ${ }^{2}$ Sensing, Imaging and Signal Processing, School of EEE, University of Manchester, Manchester, UK \\ Email: *Wuliang.Yin@manchester.ac.uk
}

How to cite this paper: Yin, W. L., York, T., \& Ozanyan, K. B. (2018). Teaching Approach and Materials for Enhancing Student Learning Experience in Tomography Engineering: Soft-Field Tomography. Creative Education, 9, 2884-2897. https://doi.org/10.4236/ce.2018.916217

Received: October 9, 2018

Accepted: December 24, 2018

Published: December 27, 2018

Copyright $\odot 2018$ by authors and Scientific Research Publishing Inc. This work is licensed under the Creative Commons Attribution International License (CC BY 4.0).

http://creativecommons.org/licenses/by/4.0/

(c) (i) Open Access

\begin{abstract}
This paper presents the development of course materials and a laboratory exercise for teaching the course "Tomography Engineering and Applications" for MSc and MEng students. The lecturing materials are enhanced by developing MATLAB codes that illustrate the working principles of finite difference and finite elements methods, allowing students to step through the algorithm development procedures and gain better insights into the taught theoretical concepts. A virtual laboratory excise is developed to allow the students to interact with image reconstruction examples by varying the controlling parameters and exam their effects. Then an assignment is designed to enhance the student learning experience by encouraging students to explore and form appreciation of the strengths and weaknesses of image reconstruction for electrical resistance tomography using EIDORS. In the process, a number of important skills in scientific research can be gained by students, i.e. make careful observations, carry out critical analysis of the results, draw sensible conclusions and report the findings in a logical manner. It is believed that these three teaching and learning components form an integral and comprehensive approach to teaching Tomography Imaging techniques at the MEng and MSc level.
\end{abstract}

\section{Keywords}

Tomography Engineering and Applications, Soft-Field Tomography, MATLAB, EIDORS 


\section{Introduction}

Originally, the term "tomography" comes from the Greek words "tomos" meaning a section and "graphy" meaning an image (Abdul Wahab et al., 2015; Beck, Dyakowski, \& Williams, 1998; Beck \& Williams, 1996). Simply, the tool of tomography is called the "tomograph" and the reconstructed image is a "tomogram". Tomography can also be defined as a process to reconstruct the image of an object of interest within the sensing zone. Since the 1970s, tomography has been widely employed in medical applications. After decades of research, tomography is also implemented in industrial applications, when it is commonly known as Industrial Process Tomography (IPT) or simply as Process Tomography (PT) (Gladden, 1997).

Due to its main advantage of being non-destructive to the process or object being measured, process tomography has become of widespread interest to researchers investigating its industrial application (Dyakowski, 1996; Qiu, Hoyle, \& Podd, 2007; Thorn, Johansen, \& Hammer, n.d.).

PT (Process Tomography) (Gladden, 1997; Wang et al., 2018) research has been pioneered in the University of Manchester since late 1980s. A unique teaching module focused on ET (Electrical Tomography) imaging techniques and applications has been offered as a module for the DISP (Digital Imaging and Signal Processing) MSc course since 2010.

Mastering this subject requires students to gain a deep understanding in a number of relevant areas, in particular, two major concepts, i.e. the forward problem and the inverse problem (Munck, Faes, \& Heethaar, 2000). The solution of the forward problem relies heavily on the understanding of Electromagnetics. Electromagnetics traditionally is not an easy subject, especially difficult for the students to establish abstract concepts such as fields, potentials, operators in two weeks of this module (Warnick, Selfridge, \& Arnold, 1997). The solution of inverse problems involves abstract matrix manipulations, which seems to relate litthe to the practical process of forming an image from boundary voltage measurements (Sarvas, 1987). Hands-on experience on practical systems would be helpful and preferable, however, ET systems are specialist instruments and expensive, therefore it is not feasible to offer a large number of students experimental-systems-based exercise.

This paper focuses on designing computer aided materials for lectures and labs to enhance student's experience in learning, including the following work:

1) MATLAB ("http://www.mathworks.com/products/matlab/", n.d.) codes were developed to demonstrate the steps and procedures in the forward solution process, and visualisation is used to display the results.

2) In addition, a virtual lab has been designed based on the EIDORS 3D toolkit that is hosted by the MATLAB programming environment to enhance students" understanding of the inverse problem without linking to specialist hardware systems. These virtual experiments help the students to appreciate the essential nature of an experiment without being confused or distracted by the 
complications of the real experimental procedures.

3) Furthermore, an assignment is designed for the student to explore further how the image quality is affected by various parameters and find the sensible range and optimal values for these parameters.

The above which combines software, hardware and algorithm together forms an integral and comprehensive approach to teaching Tomography Imaging techniques at the MEng and MSc level.

\section{Lectures}

In 10 hours lectures, the following contents are covered:

1) An overview of the soft-field tomography;

2) Electrostatic fields analysis;

3) The forward and the inverse problem;

4) Electrical tomography systems;

5) Example applications.

Fundamental concepts such as determinant, inverse in matrix operations, Faradays law, Gauss law, potential, field, flux are pre-requisites (York, 2011). However, through a quiz in the beginning of the course, it was found that only a small portion of the students understood the Div, Grad and Curl operations in vector calculus. Therefore, a strategy is adopted that numerical examples and procedures should be maximally used to illustrate the field theories behind as soon as they are introduced.

On one hand, working on black board can only illustrate problems of extremely simplistic nature (mostly one dimension problems), which seem too far detached from any realistic tomography forward problems. For example, even a simple 6 elements FEM (Finite Element Method) problem is near the limits that the chalk and talk approach can handle. On the other hand, the commercial software packages, while producing perfect solutions to a complex problem, powerful as they are, do not provide a step by step approach. They miss important intermediate steps, which are vital for students to understand the solution process. Therefore it was decided to develop customised forward solvers to allow the process of scaling up the problem to be shown, so as to present a continuous link from the basic taught theory to the complex realistic problems the theory can be applied to solve. All codes have been developed in MATLAB.

\subsection{Laplace Equation}

The governing equation for the electric field in an electrical resistance tomography is the Laplace equation, which is a type of partial differential equation (PDE) (Yorkey, Webster, \& Tompkins, 1987). In 2D cases,

$$
\nabla^{2} V=\frac{\partial^{2} V}{\partial x^{2}}+\frac{\partial^{2} V}{\partial y^{2}}=0
$$

where $V$ is the voltage and $\nabla^{2}$ The Laplace operator. There are mainly two numerical techniques that can be used to solve this type of PDEs, namely, Finite 
Difference Method (FDM) and FEM. Computer-assisted materials are developed for both methods during the course.

\subsection{Finite Difference Method}

The FDM (Binns, Lawrenson, \& Trowbridge, 1992) is a numerical method for solving partial differential equations by using finite difference to approximate derivative. Normally, the domain is discretised into a rectangular grid as shown in Figure 1 and the Laplace operator is approximated by the finite difference (Equation (2)). In Figure 1, the grid sizes in $x$ and $y$ directions are $l$ and $h$ respectively. $V$ represents voltages on the nodes. $\mathrm{i}$ and $\mathrm{j}$ are used for numbering the nodes in the $2 \mathrm{D}$ grid.

$$
\begin{aligned}
& \frac{\partial^{2} V}{\partial x^{2}}=\left(V_{i+1, j}+V_{i-1, j}-2 V_{i, j}\right) / l^{2} \\
& \frac{\partial^{2} V}{\partial y^{2}}=\left(V_{i, j+1}+V_{i, j-1}-2 V_{i, j}\right) / h^{2}
\end{aligned}
$$

Substituting Equation (2) into Equation (1) arrives at

$$
\frac{\left(V_{i+1, j}+V_{i-1, j}-2 V_{i, j}\right)}{l^{2}}+\frac{\left(V_{i, j+1}+V_{i, j-1}-2 V_{i, j}\right)}{h^{2}}=0
$$

This converts the PDEs into a set of linear equations. By solving the set of linear equations, the voltage and field distribution can be obtained. One example is shown in Figure 2. Equipotential lines shown as contours when there are a current source $(20,5)$ with a potential $10 \mathrm{~V}$ and current sink $(20,35)$ with a potential $0 \mathrm{~V}$ in a 40 by $40 \mathrm{~mm}$ rectangular space of uniform conductivity, simulating two electrodes in Electrical Inductive Tomography (EIT).

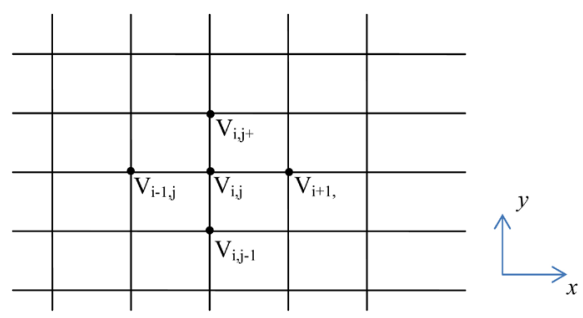

Figure 1. A finite difference grid.

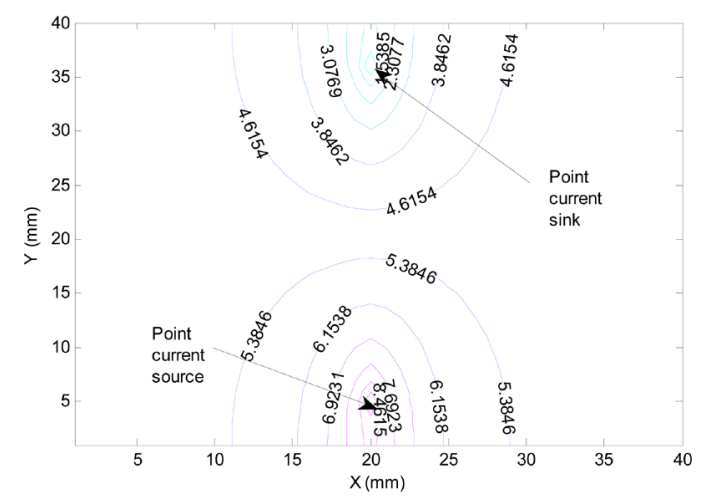

Figure 2. Voltage distributions from finite difference calculations. 


\subsection{Finite Element Methods}

FEM is another widely used numerical technique for solving the PDE which governs the electromagnetic field problem based on variational principles (Jin, 2014). The solution space is meshed into small elements-triangles in $2 \mathrm{D}$ or tetrahedral in $3 \mathrm{D}$ cases. The main advantage of the FEM over the FDM is its ability to handle objects with arbitrary boundary shapes.

A 2D FEM code was developed to demonstrate the working principle of the FEM. A simple numerical example is shown below to illustrate the procedures in FEM. Firstly, the domain is discretised into four triangular elements ((1), (2), (3) and (4)), each having three nodes as shown in Figure 3. Voltages at nodes 1 and 4 are $0 \mathrm{~V}$. Voltages at nodes 3 and 6 are $10 \mathrm{~V}$. Table 1 and Table 2 show the data structures for the element and node files.

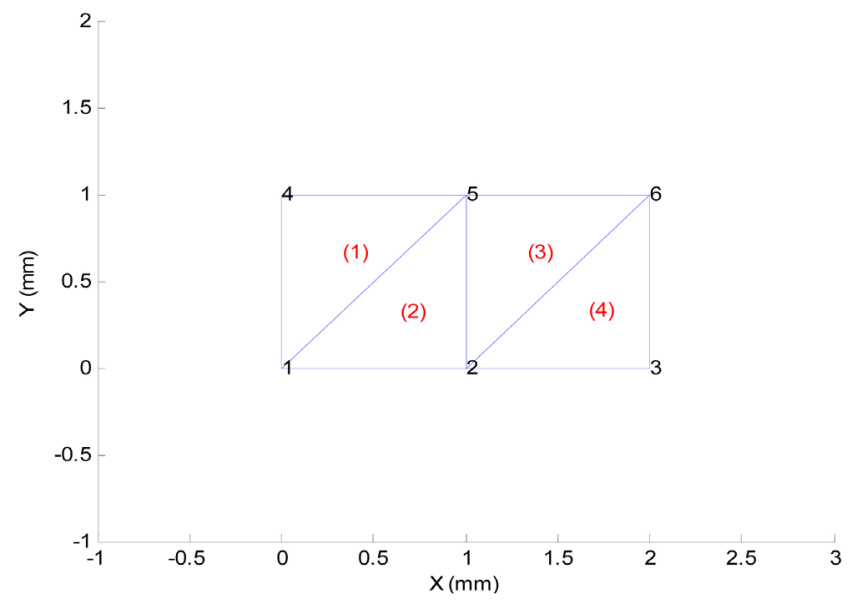

Figure 3. Meshing and labelling for a simple 2D FEM problem.

Table 1. Element data structure.

\begin{tabular}{ccccc}
\hline Element No. & Node 1 & Node 2 & Node 3 & Conductivity (S/m) \\
\hline$(1)$ & 1 & 5 & 4 & 10 \\
$(2)$ & 1 & 2 & 5 & 10 \\
$(3)$ & 2 & 6 & 5 & 10 \\
$(4)$ & 2 & 3 & 6 & 10 \\
\hline
\end{tabular}

Table 2. Node data structure.

\begin{tabular}{ccc}
\hline Node No & X coordinate & Y coordinate \\
\hline 1 & 0 & 0 \\
2 & 1 & 0 \\
3 & 2 & 0 \\
4 & 0 & 1 \\
5 & 1 & 1 \\
6 & 2 & 1 \\
\hline
\end{tabular}


To convert a PDE to a system of linear equations, the stiffness matrix must be calculated firstly. The primary characteristics of a finite element are embodied in the element stiffness matrix. The term "stiffness matrix" originally came from structural finite element domain where it indicates the resistance of the element to deformation when subjected to loading. In the case of EIT, the elements of the stiffness matrix represent conductance. The process of populating the stiffness matrix when the contributions from elements ((1) to (4)) are added one by one is shown in Figure 4. For example, the first triangular element constitutes 3 nodes (nodes 1, 4 and 5); it contributes to the stiffness matrix in entries S11, S14, S41, S44, S45, S54, and S55. Similarly, the contribution of the second element with nodes 1, 2 and 5 has effects on S11, S12, S21, S22, S25, S52 and S55.

Once the stiffness matrix is populated, then voltages can be obtained by solving a system of linear equations, and the solution can be displayed in MATLAB, as shown in Figure 5.

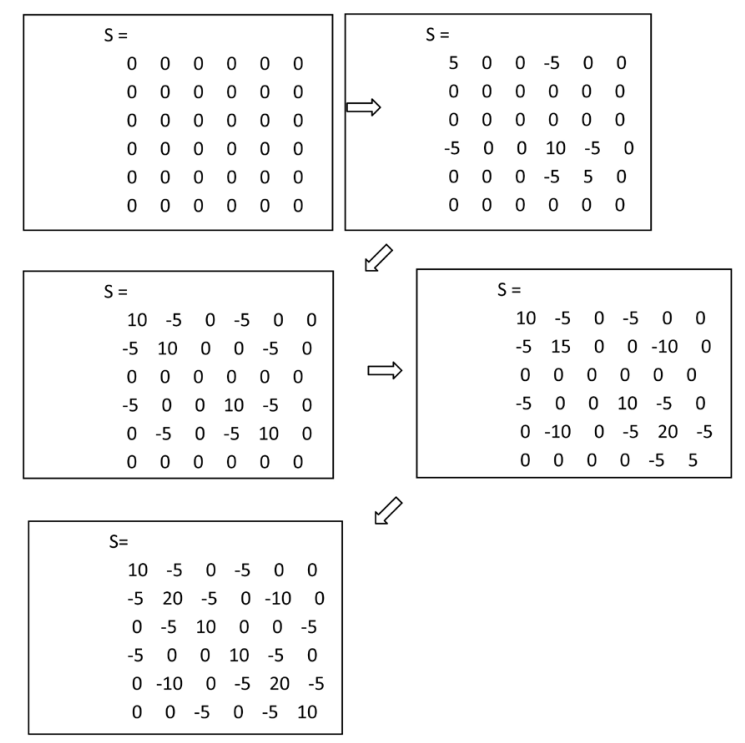

Figure 4. The evolution of stiffness matrix.

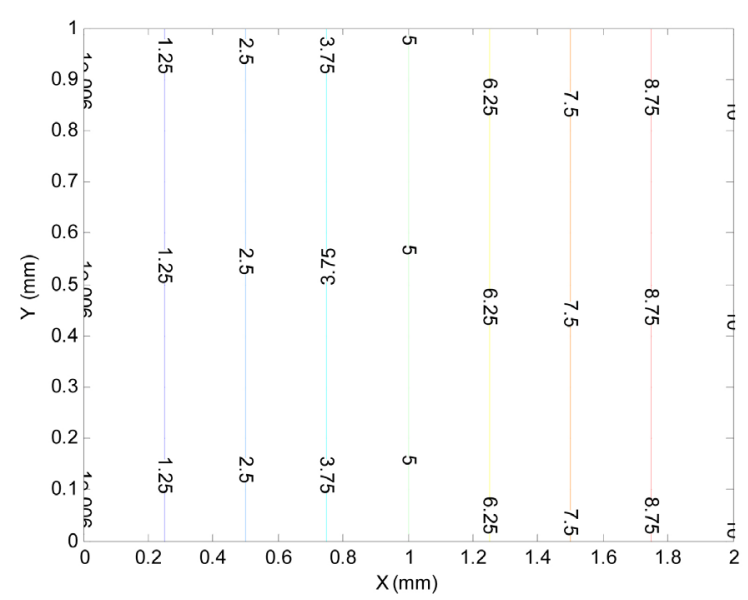

Figure 5. Equipotential lines for the simple FEM example in Figure 3. 
At this stage, students should get a step by step understanding of how a simple forward problem can be solved following FEM procedures. Next, an appreciation of how this code can be used to solve realistic ERT (Electrical Resistance Tomography) problems is demonstrated as shown in Figure 6. Figure 7 shows the voltage distributions when currents are injected from nodes 1 and 16 simulating point sources, which are indicated in Figure 6 and colour bar indicates voltages. This can be achieved by using the same code, replacing the element and node files and assigned nodes to different potentials to simulating excitation and receiving electrodes.

\section{The Laboratory}

The aim of the laboratory is to enhance the soft field learning experience by introducing students to the detailed process of image reconstruction for electrical tomography, from generation of a model through, meshing, computing the forward and inverse solutions, and visualisation (Yin, 2013; York, 2011).

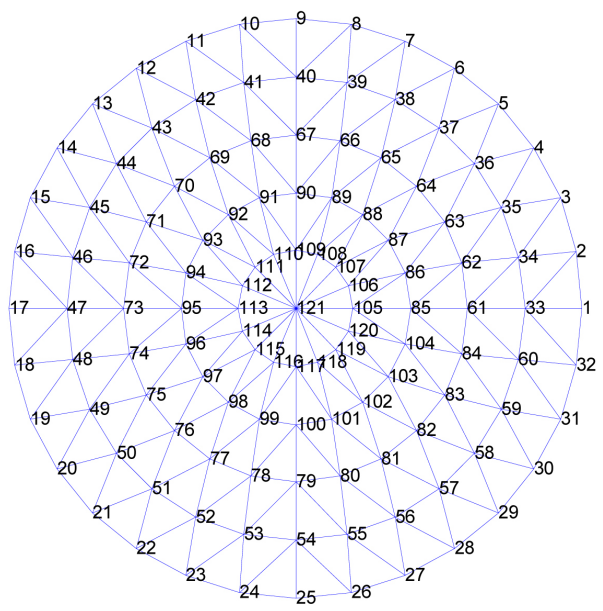

Figure 6. A mesh with node numbers annotated for ERT.

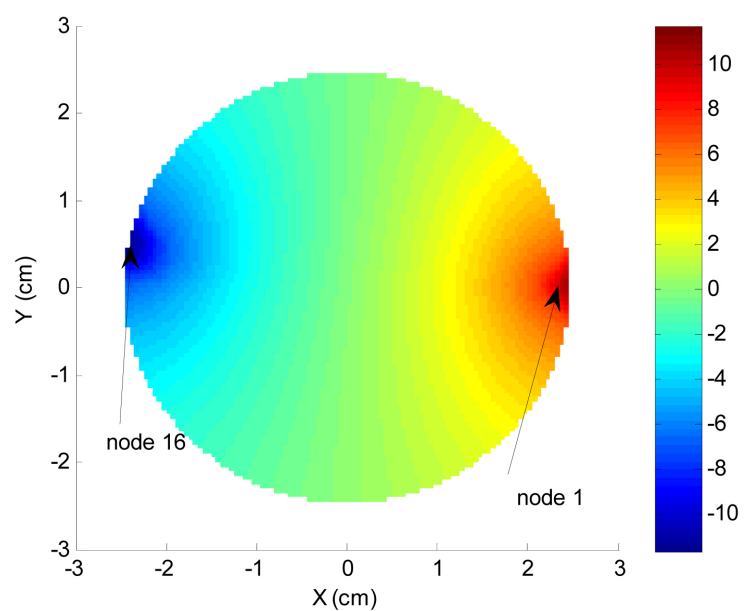

Figure 7. Voltage distributions when currents are injected from nodes 1 and 16 simulating point sources. 


\subsection{Software}

The practical work is based on the EIDORS 3D toolkit (Adler \& Lionheart, 2006; Polydorides \& Lionheart, 2002; Vauhkonen, Lionheart, Heikkinen, Vauhkonen, \& Kaipio, 2001) that is hosted by the MATLAB programming environment. MATLAB is a high-level technical computing language and interactive environment for algorithm development, data visualization, data analysis, and numeric computation. It is relatively slow to compute solutions compared to, for instance, $\mathrm{C}, \mathrm{C}++$, and Fortran. However, it provides an environment that encourages rapid prototyping of algorithms and exploration of applications through the use of a matrix-based structure.

EIDORS (“http://eidors3d.sourceforge.net/", n.d.) (Electrical Impedance and Diffuse Optical Reconstruction Software) has been developed to provide freely available tools that can be used to reconstruct electrical or optical material properties from boundary measurements. Nonlinear and ill-posed problems such as electrical impedance and optical tomography are typically approached using a finite element model for the forward calculations and a regularized nonlinear solver for obtaining a unique and stable inverse solution. Most of the commercially available finite element programs are unsuitable for solving these problems because of their conventional inefficient way of calculating the Jacobian, and their lack of accurate electrode modelling. A complete package for the two-dimensional EIT problem was officially released by Vauhkonen et al. in 2000. However most industrial and medical electrical imaging problems are fundamentally three-dimensional and EIDORS 3D was released in 2004. To assist development a free toolkit of MATLAB routines which can be employed to solve the forward and inverse EIT problems in three dimensions has been released.

The EIDORS environment essentially consists of a number of functions that are loaded and run in the MATLAB environment according to the demands of each application and preferences of each user. The original functions can be found on the EIDORS websites.

\section{NETGEN}

("http://sourceforge.net/apps/mediawiki/netgen-mesher/index.php?title=Main Page", n.d.) which is an automatic mesh generation tool written in $\mathrm{C}++$ capable of generating meshes in two and three dimensions, is also used in this course. The program is open source and is available for Unix/Linux, Windows $\mathrm{NT} / \mathrm{XP} / \mathrm{Vista}$ as well as the MacOS platforms for both 32-bit and 64-bit architectures. Netgen generates triangular or quadrilateral meshes in $2 \mathrm{D}$, and tetrahedral meshes in $3 \mathrm{D}$.

\subsection{Laboratory Exercise}

The laboratory exercise includes following procedures:

1) Create a model for the vessel;

2) Create a mesh using Netgen and import the mesh to EIDORS;

3) Forward calculation: compute the predicted boundary voltages, compute 
the Jacobian;

4) Import the measurement file;

5) Compute the reconstructed images and display the images.

To carry out an activity, students need to manually call each function in EIDORS and then specify parameters through either interactive GUI or text input. The parameters of interest are mainly the number of iteration and the smoothing factors, which have significant effects on the images obtained. An excessively large smoothing factor tends to blur sharp features in the image while an excessively small smoothing factor would not eliminate noise effectively and produce artificial features. An adequate number of iterations is needed for the reconstructed measurement error to reduce to a tolerant level, while excessive iteration would cause the solver to diverge. It is in fact one of the main aims of this exercise to let students have an understanding and appreciation of this concept-useful images can only be produced with sensible ranges of these parameters.

The vessel that the laboratory used as a phantom is shown, together with some example images in Figure 8. 16 electrodes are evenly distributed around the vessel and the objects to be imaged are plastic and metallic rods in a tap water background with a nominal conductivity of $0.1 \mathrm{mS} / \mathrm{cm}$. The vessel is made of Perspex and the electrodes of stainless steel. The diameters of the plastic and metallic rods are $1.5 \mathrm{~cm}$.

An iterative MATLAB function is used to record the tank model parameters such as, tank radius, height, shape, size and number of electrodes, the number of planes and its position in height. Cross-sectional images then can be produced following the remaining steps in the lab script, which include importing mesh, computing the forward solution, computing the inverse solution and image display (York, 2011). The colours in the reconstructed cross-section images represent as shown in Table 3.

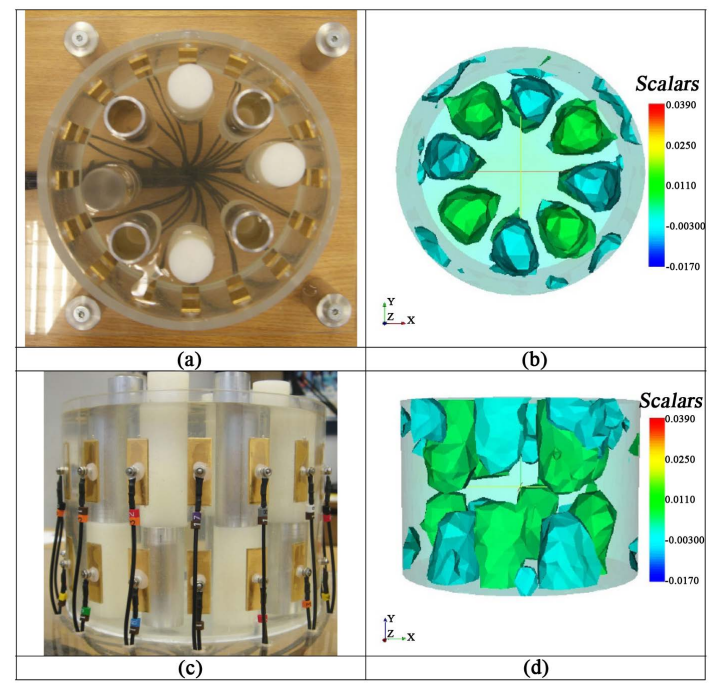

Figure 8. The Vessel. (a) top view showing object distribution; (b) top view of the reconstruction; (c) side view of the vessel; (d) side view of the reconstruction. 
Table 3. Colours in the reconstructed cross-section images.

\begin{tabular}{ccc}
\hline Tap water & Higher conductivity & Lower conductivity coordinate \\
\hline green & red & blue \\
\hline
\end{tabular}

\section{The Assignment}

The assignment is intended to encourage students to explore and form appreciation of the strengths and weaknesses of image reconstruction for electrical resistance tomography using EIDORS. Each student is given a measurement reference file and measurement data files to perform image reconstruction using the iterative Gauss Newton methods (Yorkey et al., 1987) and to determine preferred values for the reconstruction parameters, i.e. smoothing (regularization) factors and number of iterations with some prior knowledge such as the size, shape and materials of the objects. A reference file contains the measured voltages when the vessel was filled with saline (homogenous distribution of conductivity). Measurements data files contain measurements when there were objects inside the vessel.

The quality of reconstructed images strongly depends on the regularization/smoothing parameter, therefore, it is crucial to choose an optimal value range, so that a reasonable solution can be obtained. In general, a small value of the smoothing factor preserve higher spatial frequency components but the influence of noises may make the solution physically unacceptable. Conversely, a large value of suppresses the data noises but increases the smoothness of the image. The number of iteration is as important as the smoothing factor; small number of iteration produces blurred images while excessive iteration cause the solution to diverge, resulting in meaningless images. In most all cases, the optimum range is problem specific and should be found empirically.

The report should cover what have been done, what have been observed and some conclusions in a conference paper format. The report is in the form a conference paper and this is to give the students an opportunity to appreciate how a piece of scientific work should be carried out and reported in normal academic practise.

Students have two days to complete the assignment, including performing image reconstruction and analysis of the data files using EIDORS, and writing the report. They are advised to divide their time equally between using EIDORS and writing the report.

This kind of task is the first of its kind for most students. It is expected that they gain a number of important skills in scientific research, i.e. make careful observations, carry out critical analysis of the results, draw sensible conclusions and report the findings in a logical manner.

The assignment is arranged after the laboratory, so students should already be familiar with EIDORS and the process of image reconstruction. Most students are able to produce images, however, the main difference between those who achieved high and low marks is the quality of the analysis and writing. 
Figure 9 is an example from a student report exploring the effect of regularization parameters and the number of iterations on the reconstructed images. Results are displayed as colour-coded cross-sections. Red colour denotes regions of high conductivities while blue colour represents that of low conductivities.

With accompanying texts explaining the results:

"The grid of results is arranged into rows and columns of increasing iterations and smoothing factors respectively. Higher smoothing factor results in the removal of higher spatial frequency components, while lower smoothing factor preserve them. Taking iteration number of 5 as an example, the effect is drastic and at the extremes it either results in lots of high frequency or almost no distinguishable objects with a smooth gradient across the whole image. The effect of iterations on the images is as drastic as the smoothing; focusing on the images with 1e-5 smoothing factor, less than 5 iterations results in some identifiable objects with very apparent noise at the edges. More than 10 iterations reduce noise but the spatial resolution is poor and some objects fade away. It seems from these reconstructions that a smoothing factor of $1 \mathrm{e}-5$ with the number of iterations 5 produces the optimum results."

This student correctly identified the appropriate range for the smoothing factor $1 \mathrm{e}-5$ and the number of iteration 5 . With this set of parameter, two metallic and one Perspex objects are clearly seen. The actual positions for this set of data is in Figure 10.

However, for a small number of students, they did not find the suitable range for the reconstruction parameters, so that no reasonable results were obtained.

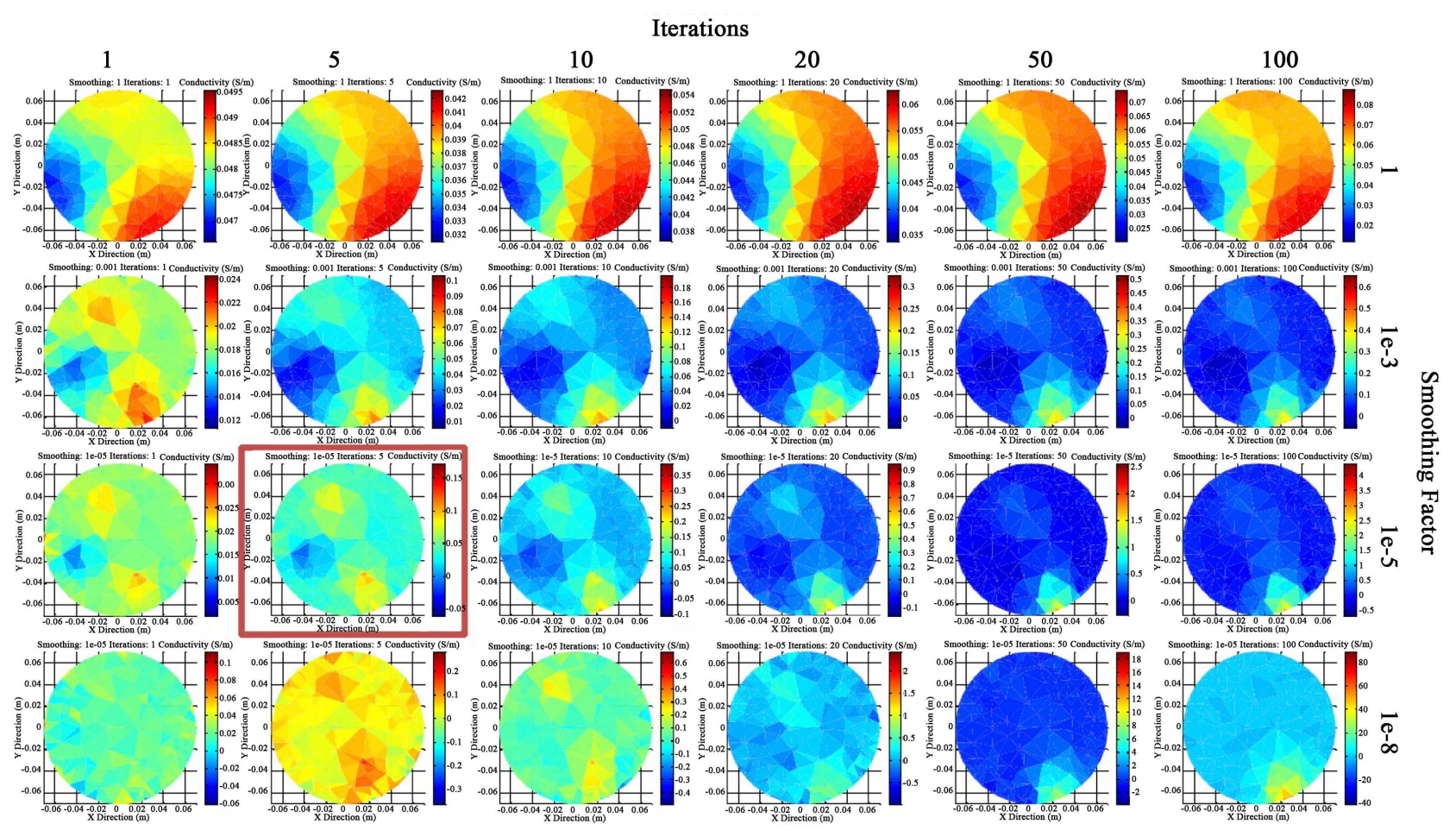

Figure 9. The effect of smoothing factors $(1,1 \mathrm{e}-3,1 \mathrm{e}-5 \& 1 \mathrm{e}-8)$ and iterations $(1,5,10,20,50,100)$ on the reconstructed images. 


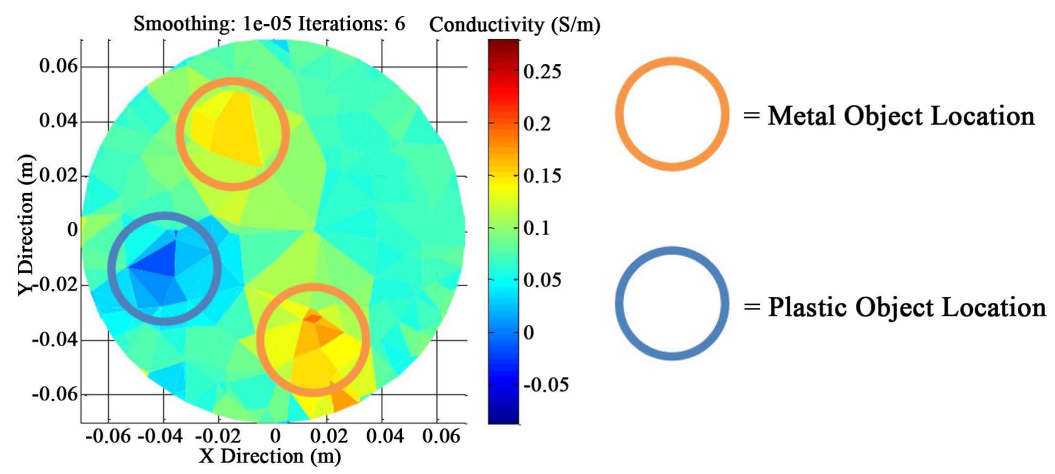

Figure 10. Correct position for reconstructions in Figure 9.

For example, one student started from iteration number of 10 and increase in a step of 10 to 100 . A suitable range is normally empirically determined in practice. In this assignment, prior knowledge such as the sizes and material properties of the objects can be used to visually evaluate the reconstructed results. A more rigorous approach would be the L curve method (Calvetti, Morigi, Reichel, \& Sgallari, 2000), which is out of the scope of this assignment.

One cause for the poor performance of students in the assignment were the low quality of the measurements data, which even in the suitable range of the reconstruction parameters give blurred images. In addition, different measurement files had to be given to different students to ensure they work independently; however, some data sets are less noisy than others, which pose challenges for fair marking. Only one student out 40 has found a way of quantitatively assessing the image quality by analyzing image histograms.

\section{Conclusion}

We have discussed the development the course materials for the MSc course-tomography engineering and applications-that has run for three years at Manchester. This has been designed and developed primarily based on MATLAB and EIDORS. During the lectures, MATLAB codes are used to illustrate the working principles of finite difference and finite elements methods, allowing students to step through the algorithm development procedures. A laboratory excise is developed to allow the students to interact with image reconstruction examples by varying the controlling parameters and exam their effects. Then an assignment is designed to enhance the student learning experience by encouraging students to explore and form appreciation of the strengths and weaknesses of image reconstruction for electrical resistance tomography using EIDORS. It is believed that the above form an integral and comprehensive approach to teaching Tomography Imaging techniques at the MEng and MSc level.

\section{Conflicts of Interest}

The authors declare no conflicts of interest regarding the publication of this paper. 


\section{References}

Abdul Wahab, Y., Abdul Rahim, R., Fazalul Rahiman, M. H., Ridzuan Aw, S., Mohd Yunus, F. R., Goh, C. L. et al. (2015). Non-Invasive Process Tomography in Chemical Mixtures-A Review. Sensors and Actuators B: Chemical, 210, 602-617. https://doi.org/10.1016/j.snb.2014.12.103

Adler, A., \& Lionheart, W. R. B. (2006). Uses and Abuses of EIDORS: An Extensible Software Base for EIT. Physiological Measurement, 27, S25. https://doi.org/10.1088/0967-3334/27/5/S03

Beck, M. S., \& Williams, R. A. (1996). Process Tomography: A European Innovation and Its Applications. Measurement Science and Technology, 7, 215. https://doi.org/10.1088/0957-0233/7/3/002

Beck, M. S., Dyakowski, T., \& Williams, R. A. (1998). Process Tomography-The State of the Art. Transactions of the Institute of Measurement and Control, 20, 163-177. https://doi.org/10.1177/014233129802000402

Binns, K. J., Lawrenson, P. J., \& Trowbridge, C. W. (1992). The Analytical and Numerical Solution of Electric and Magnetic Fields. Chichester: Wiley.

Calvetti, D., Morigi, S., Reichel, L., \& Sgallari, F. (2000). Tikhonov Regularization and the L-Curve for Large Discrete Ill-Posed Problems. Journal of Computational and Applied Mathematics, 123, 423-446. https://doi.org/10.1016/S0377-0427(00)00414-3

Dyakowski, T. (1996). Process Tomography Applied to Multi-Phase Flow Measurement. Measurement Science and Technology, 7, 343. https://doi.org/10.1088/0957-0233/7/3/015

Gladden, L. F. (1997). Process Tomography: Principles, Techniques and Applications. Measurement Science and Technology, 8. https://doi.org/10.1088/0957-0233/8/4/021

http://eidors3d.sourceforge.net/

http://sourceforge.net/apps/mediawiki/netgen-mesher/index.php?title=Main_Page

http://www.mathworks.com/products/matlab/

Jin, J. (2014). The Finite Element Method in Electromagnetics (3rd ed.). Hoboken, NJ: Wiley.

Munck, J. C. de, Faes, T. J. C., \& Heethaar, R. M. (2000). The Boundary Element Method in the Forward and Inverse Problem of Electrical Impedance Tomography. IEEE Transactions on Biomedical Engineering, 47, 792-800. https://doi.org/10.1109/10.844230

Polydorides, N., \& Lionheart, W. R. B. (2002). A Matlab Toolkit for Three-Dimensional Electrical Impedance Tomography: A Contribution to the Electrical Impedance and Diffuse Optical Reconstruction Software Project. Measurement Science and Technology, 13, 1871. https://doi.org/10.1088/0957-0233/13/12/310

Qiu, C., Hoyle, B. S., \& Podd, F. J. W. (2007). Engineering and Application of a Dual-Modality Process Tomography System. Flow Measurement and Instrumentation, 18, 247-254. https://doi.org/10.1016/j.flowmeasinst.2007.07.008

Sarvas, J. (1987). Basic Mathematical and Electromagnetic Concepts of the Biomagnetic Inverse Problem. Physics in Medicine \& Biology, 32, 11.

https://doi.org/10.1088/0031-9155/32/1/004

Thorn, R., Johansen, G. A., \& Hammer, E. A. (n.d.). Three-Phase Flow Measurement in the Offshore Oil Industry Is There a Place for Process Tomography (p. 8).

Vauhkonen, M., Lionheart, W. R. B., Heikkinen, L. M., Vauhkonen, P. J., \& Kaipio, J. P. (2001). A MATLAB Package for the EIDORS Project to Reconstruct Two-Dimensional 
EIT Images. Physiological Measurement, 22, 107. https://doi.org/10.1088/0967-3334/22/1/314

Wang, H. G., Che, H. Q., Ye, J. M., Tu, Q. Y., Wu, Z. P., Yang, W. Q., \& Ocone, R. (2018). Application of Process Tomography in Gas-Solid Fluidised Beds in Different Scales and Structures. Measurement Science and Technology, 29, Article ID: 044001. https://doi.org/10.1088/1361-6501/aaa509

Warnick, K. F., Selfridge, R. H., \& Arnold, D. V. (1997). Teaching Electromagnetic Field Theory Using Differential Forms. IEEE Transactions on Education, 40, 53-68. https://doi.org/10.1109/13.554670

Yin, W. (2013). Lab Script for Tomography Engineering and Application-Soft Field Tomography.

York, T. (2011). Lecture Notes for Tomography Engineering and Application-Soft Field Tomography.

Yorkey, T. J., Webster, J. G., \& Tompkins, W. J. (1987). Comparing Reconstruction Algorithms for Electrical Impedance Tomography. IEEE Transactions on Biomedical Engineering, 34, 843-852. https://doi.org/10.1109/TBME.1987.326032 